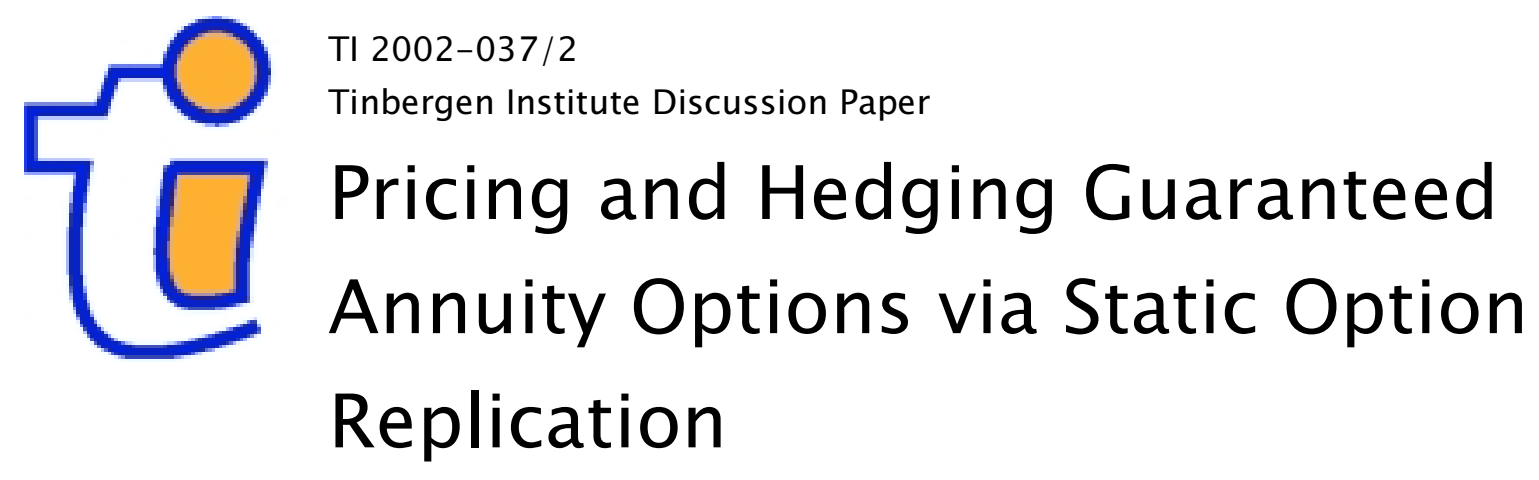

Antoon Pelsser

Actuarial Dept., Nationale Nederlanden, Rotterdam, Faculty of Economics, Erasmus University Rotterdam, and Tinbergen Institute 
Tinbergen Institute

The Tinbergen Institute is the institute for economic research of the Erasmus Universiteit Rotterdam, Universiteit van Amsterdam and

Vrije Universiteit Amsterdam.

Tinbergen I nstitute Amsterdam

Keizersgracht 482

1017 EG Amsterdam

The Netherlands

Tel.: +31.(0)20.5513500

Fax: $\quad+31 .(0) 20.5513555$

Tinbergen Institute Rotterdam

Burg. Oudlaan 50

3062 PA Rotterdam

The Netherlands

Tel.: $\quad+31 .(0) 10.4088900$

Fax: $\quad+31 .(0) 10.4089031$

Most TI discussion papers can be downloaded at

http://www.tinbergen.nl 


\title{
Pricing and Hedging Guaranteed Annuity Options via Static Option Replication ${ }^{1}$
}

\author{
Antoon Pelsser \\ Head of ALM Dept \\ Nationale-Nederlanden \\ Actuarial Dept \\ PO Box 796 \\ 3000 AT Rotterdam \\ The Netherlands \\ Tel: (31)10 - 5139485 \\ Fax: (31)10 - 5130120 \\ E-mail: antoon.pelsser@nn.nl

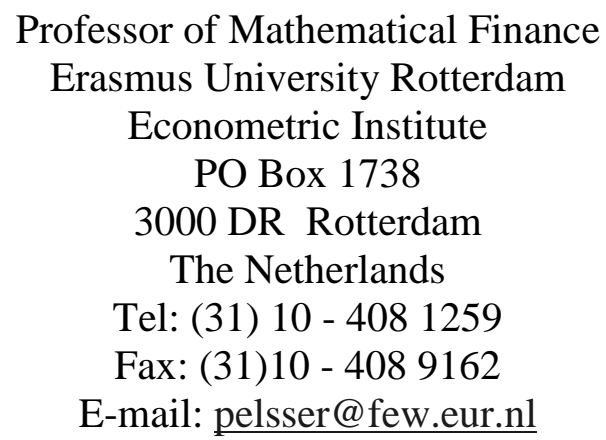

First version: January 2002

This version: 12-Mar-2002 13:27

1 This article expresses the personal views and opinions of the author. Please note that ING Group or Nationale-Nederlanden neither advocates nor endorses the use of the valuation techniques presented here for its external reporting. The author would like to thank Pieter Bouwknegt for valuable insights and comments. 


\title{
Pricing and Hedging Guaranteed Annuity Options via Static Option Replication
}

\begin{abstract}
In this paper we derive a market value for Guaranteed Annuity Option using martingale modelling techniques. Furthermore, we show how to construct a static replicating portfolio of vanilla interest rate swaptions that replicates the Guaranteed Annuity Option. Finally, we illustrate with historical UK interest rate data from the period 1980 until 2000 that the static replicating portfolio is extremely effective as a hedge against the interest rate risk involved in the GAO, that the static replicating portfolio is considerably cheaper than up-front reserving and also that the replicating portfolio provides a much better level of protection than an up-front reserve.
\end{abstract}

JEL Codes: G13, G22 


\section{Introduction}

Recently, considerable publicity is drawn to life-insurance policies with Guaranteed Annuity Options (GAO's). Equitable, a large British insurance office, had to close for new business as a portfolio of old insurance policies with GAO's became an uncontrollable liability. In this paper we want to propose a hedging methodology that can help insurance companies to avoid such problems in the future.

During the last few years, many authors have applied no-arbitrage pricing theory from financial economics to calculate the value of embedded options in (life-)insurance contracts. Initially, the work was focussed on valuing return guarantees embedded in equity-linked insurance policies, see for example Brennan and Schwartz (1976), Boyle and Schwartz (1977), Aase and Persson (1994), Boyle and Hardy (1997) and Bacinello and Persson (2002). In equity-linked contracts, the minimum return guarantee can be identified as an equity put option, and hence the "classical" Black-Scholes (1973) option pricing formula can be used to determine the value of the guarantee.

More life-insurance policies are not explicitly linked to the value of a reference equity fund. Traditionally, life-insurance policies promise to pay a nominal amount of money to the policyholder at expiration of the contract. In order to compensate the policyholder for the relatively low base-rates which are used for premium calculation, various profit-sharing schemes have been employed by insurance companies. Through a profit-sharing scheme, part of the excess return (i.e. return on investments above the base rate) that the insurance company makes is being returned to the policyholders. However, since only the excess return is being shared with the policyholders and not the shortfall, having a profit-sharing scheme in place is equivalent to giving a minimum return guarantee (at the level of the base rate) to the policyholders. This type of embedded return guarantees has only recently been analysed in the literature, see for example Aase and Persson (1997), Grosen and Jørgensen (1997), (2000a) and (2002), Miltersen and Persson (1999) and (2000) and Bouwknegt and Pelsser (2002).

Guaranteed Annuity Options are another example of minimum return guarantees, but in the case of GAO's the guarantee takes the form of the right to convert an assured sum into a life annuity at the better of the market rate prevailing at the time of conversion and a guaranteed rate. Many lifeinsurance companies in the UK issued pension-type policies with GAO's in the 1970's and 1980's. During this time UK interest rates were very high, above 10\% between 1975 and 1985. 
Hence, adding GAO's with implicit guaranteed rates around $8 \%$ was considered harmless at that time due to the fact that these option were so far "out-of-the-money". Due to the fall of UK interest rates far below 8\% (currently UK interest rates are at a level of 5\%), the GAO's have become an uncontrollable liability which caused the downfall of Equitable in 2000. The issue of determining the value of GAO's has been addressed recently by Yang (2001), Lee (2001) and Balotta and Haberman (2002).

As is evident from the literature overview provided here, the main focus has been given to determining the value of embedded options. With the downfall of Equitable it has, in our view, become apparent that not only the valuation should be addressed, but also the hedging of embedded options. Although the hedging issue seems trivial at first sight: any derivative can be replicated by executing a delta-hedging strategy. However, the options written by insurance companies have such long maturities and the insured amounts are so high that executing a deltahedging strategy can have disastrous consequences.

Typically, an insurance company has sold put options to its policy holders. To create a deltaneutral position the insurance company has to buy the underlying asset of the put option. If markets fall, the insurance company has to sell off part of its asset position to remain delta-neutral. This will create more downside pressure on the asset prices, especially if the insurance company is trying to rebalance a large position. Hence, executing a delta-hedging strategy for a short put position can create dangerous "feedback loops" in financial markets which can have disastrous consequences. Similar feedback loops were present in Portfolio Insurance strategies which used delta-hedging to create synthetic put options and were very popular during the 1980's. Automated selling orders generated by computers trying to follow blindly the delta-hedging strategy have been blamed for triggering the October 1987 crash. After the 1987 crash, Portfolio Insurance strategies very quickly lost their appeal and are hardly used nowadays. A second complication with executing a delta-hedging strategy is that delta hedging required frequent rebalancing of the hedging assets in order to remain delta-neutral. Especially for long maturity options, this can be quite expensive because of the transactions costs involved.

We want to propose the use of static option replication as a viable alternative for insurance companies to hedge their embedded options. A static option replication can be set up if a portfolio of actively traded options can be found that (approximately) replicates the payoff of the derivative under consideration. Once the payoff of the derivative has been replicated, the no-arbitrage 
condition implies that also for all prior times the value of the derivative is replicated by the static portfolio. Static replication hedging techniques for exotic equity options have been introduced by Derman, Ergener and Kani (1995) and Carr, Ellis and Gupta (1998). The advantages of static replication are obvious: once the initial static hedge has been set up, no rebalancing is needed in order to keep the derivative hedged. In practice, it is not always possible to find a set of actively traded options that perfectly replicates the payoff of a given derivative. However, if the approximation is close enough the static replication portfolio will track the value of the derivative under a wide range of market conditions.

In this paper we want to show how Guaranteed Annuity Options can be statically replicated using a portfolio of vanilla interest rate swaptions. Interest rate swaptions are actively traded for a wide variety of maturities and single trades can be executed for large notionals amounts. Using the history of UK interest rates, we demonstrate that a judiciously chosen static portfolio of swaptions can hedge GAO's over a long time horizon and under a wide range of market conditions. Hence, we illustrate that static replication offers a realistic possibility for insurance companies to hedge their exposure to embedded options in their portfolios.

The remainder of this paper is organised as follows. In Section 2 we describe the payoff of Guaranteed Annuity Options and we derive a pricing formula using martingale modelling. In Section 3 we construct the static replication portfolio consisting of vanilla swaptions. In Section 4 we illustrate the effectiveness of the static portfolio with a historic test using UK interest rate data from 1980 until 2000. Finally, we conclude in Section 5.

\section{Guaranteed Annuity Options}

Let us consider the market value of annuities at the moment when they are bought. An annuity is financed by a single premium, in our case this single premium equals the lump sum payment of the capital policy. Suppose the annuity is bought at time $T$ by a person of age $x$. Conditional on the survival probabilities ${ }_{n} p_{x}$ from the mortality table we can write the market value of the annuity $\ddot{a}_{x}(T)$ with an annual payment of 1 as

$$
\ddot{a}_{x}(T)=\sum_{n=0}^{\omega-x}{ }_{n} p_{x} D_{T+n}(T),
$$


where ${ }_{n} p_{x}$ denotes the probability that an $x$ year old person survives $n$ years and $D_{T+n}(T)$ denotes the market value at time $T$ of a discount factor with maturity $T+n$. Also note that, the sum is truncated at age $\omega$, the maximum age in the mortality table. Given the market value $\ddot{a}_{x}(T)$, the market annuity payout rate $r_{x}(T)$ over an initial single premium of 1 is given by

$$
r_{x}(T)=1 / \ddot{a}_{x}(T)
$$

This approach to market values is fully consistent with the construction of a portfolio of default free bonds that match exactly the expected cash flows of the annuity.

Note, that we assume that the lump sum payment $L$ at time $T$ is a deterministic quantity. This may seem inconsistent with the fact that GAO's have been issued on unit-linked contracts and withprofit contracts, because in these types of contracts the value of the capital policy at time $T$ is unknown. For unit-linked contracts this is definitely true, and in a recent paper by Ballotta and Haberman (2002) they attempt to address this issue. ${ }^{2}$ On the other hand, many of the policies offered, especially the policies of Equitable, are with-profits policies. In this case, there is a minimum capital payment $L$ that is known to be paid out at time $T$. Via the profit-sharing mechanism, the amount $L$ can only increase. Hence, each year of profit-sharing will add an additional "layer" to the contract with an additional GAO. But this implies that the analysis we offer in this paper is valid for with-profits policies, since each layer of profit-sharing can be valued and hedged independently.

Suppose that an $x$ year old policyholder has an amount of money $L$ at his disposal at time $T$ which is the payout of his capital policy. The GAO option gives the policyholder the right to choose either an annual payment of $L r_{x}(T)$ based on the current market rates (see formula (2.2)) or an annual payment $L r_{x}^{G}$ using the Guaranteed Annuity $r_{x}^{G}$. A rational policyholder will select the highest annuity payout given the current term structure of interest rates. Therefore, we can rewrite the value of the GAO at the exercise date $T$ as

\footnotetext{
2 Note that Ballotta and Haberman (2002) use the a single Brownian Motion that drives the uncertainty in both the equity fund and the Heath-Jarrow-Morton (1992) model. Due to this modelling assumption, it is still the case that all the uncertainty in the GAO is essentially only interest rate driven. Hence, their paper offers no generalisation over the results derived in this paper.
} 


$$
\begin{aligned}
& L \max \left(r_{x}^{G}, r_{x}(T)\right) \Sigma_{n} p_{x} D_{T+n}(T)= \\
& L\left(r_{x}(T) \Sigma_{n} p_{x} D_{T+n}(T)\right)+L \max \left(r_{x}^{G}-r_{x}(T), 0\right) \Sigma_{n} p_{x} D_{T+n}(T)= \\
& L+L \max \left(r_{x}{ }^{G}-r_{x}(T), 0\right) \ddot{a}_{x}(T)
\end{aligned}
$$

Hence, the market value of the GAO policy at the exercise date is equal to the lump sum payment $L$ plus $L$ times the value of the GAO put-option.

In the remainder of this paper we will focus only on the value $V^{G}$ of the GAO put-option

$$
V^{G}(T)=\max \left(r_{x}^{G}-r_{x}(T), 0\right) \ddot{a}_{x}(T)
$$

To calculate the market value $V^{G}(0)$ of the GAO put-option today at time 0 , we can proceed along several paths. The uncertainty about the value of the option is due to the fact that the discount factors $D_{S}(T)$ at time $T$ are unknown quantities at time 0 . One possible approach therefore, is to model the complete term-structure of interest rates with a term-structure model, like the HeathJarrow-Morton (1992) model, to obtain an option value. The disadvantage of such an approach is that the option price cannot be determined analytically; results have to be obtained through numerical approximations which provide us with relatively little insight in the behaviour of the GAO.

To obtain a better handle on the behaviour of the GAO, we draw an analogy between the GAO and a bond option. A bond option gives the holder of the option the right, but not the obligation, to buy a bond for a given price. As the value of a bond depends on the term-structure of interest rates, we could use a term-structure model to determine the value of the bond option. In the case of a bond, all uncertainty about the term-structure of interest rates is reflected in a single quantity: the price of the bond. Hence, the value of a bond option can be determined more direct by modelling the bond-price itself as a stochastic process. This is exactly the approach that bondoption traders adopt to calculate the prices of bond-options with the Black (1976) formula.

In the case of the GAO put-option, all the uncertainty about the term-structure of interest rates is reflected in the market annuity payout rate $r_{x}(T)$. Hence, if we model the market annuity payout $r_{x}$ directly as a stochastic process, we have sufficient information to price the GAO option. The approach of using market rates, such as LIBOR rates and swap rates, has been applied in recent 
years with great success to term-structure models. This type of models, which have become known as market models, was introduced independently by Miltersen, Sandmann and Sondermann (1997), Brace, Gatarek and Musiela (1997) and Jamshidian (1998).

The main mathematical result on which this modelling technique is based is the martingale pricing theorem which states that, given a numeraire (i.e. a reference asset that is used as a new basis to express all prices in the economy in terms of this asset), an economy is arbitrage-free and complete if and only if there exists a unique equivalent probability measure such that all numeraire rebased price processes are martingales under this measure. For a proof of the martingale pricing theorem and general introduction into the mathematics involved we refer to Musiela and Rutkowski (1997); the books by Hunt and Kennedy (2000) and Pelsser (2000) focus more explicitly on interest rate derivatives.

In the economy we are considering, the traded assets are the discount bonds $D_{S}$ for the different maturities $S$. A particular convenient choice of the numeraire for the GAO put-option is the annuity $\ddot{a}_{x}=\Sigma{ }_{n} p_{x} D_{T+n}$. Note, that under the assumption that the survival probabilities ${ }_{n} p_{x}$ are deterministic, this is a portfolio of traded assets (the discount bonds) and hence a permissible choice as numeraire.

The annuity payout $r_{x}(T)$ rate for time $T$ was defined in (2.2). At times $t$ prior to $T$ we can consider the value of the portfolio of discount bonds that replicates the cash flows of an annuity starting from $T$. A person that will be $x$ years old at time $T$, has at time $t$ an age of $x$-(T-t). Hence, the market value at time $t$ of a forward annuity starting at $T$ is given by

$$
\sum_{n=0}^{\omega-x}{ }_{n+(T-t)} p_{x-(T-t)} D_{T+n}(t)=_{(T-t)} p_{x-(T-t)} \sum_{n=0}^{\omega-x}{ }_{n} p_{x} D_{T+n}(t)=_{(T-t)} p_{x-(T-t)} \ddot{a}_{x}(t)
$$

where we have used the actuarial identity ${ }_{n+m} p_{x}={ }_{m} p_{x}{ }_{n} p_{x+m}$ (see, e.g., Bowers et al. (1997), Chapter 3).

At time $t$, an insurance company can finance the forward annuity by borrowing money from time $t$ until time $T$. Only in the cases the insured survives until time $T$, will the insurance company have to repay the loan. Hence, the market value at time $t$ of this loan is given by 


$$
{ }_{(T-t)} p_{x-(T-t)} D_{T}(t)
$$

Combining equations (2.5) and (2.6), we can define the forward annuity rate as

$$
r_{x}(t)=D_{T}(t) / \ddot{a}_{x}(t)
$$

where we see that the survival probability factor ${ }_{(T-t)} p_{x-(T-t)}$ in the numerator and the denominator has cancelled. Note, that if $t=T$ this definition coincides with (2.2) since $D_{T}(T) \equiv 1$. Also note that the forward annuity rate $r_{x}$ is the numeraire rebased price of the discount bond $D_{T}$ using the numeraire $\ddot{a}_{x}$.

The martingale pricing theorem can now be applied as follows. Under the assumption that the interest rate economy admits no arbitrage opportunities, the martingale pricing theorem states that there exists a martingale probability measure $\mathbf{Q}^{\mathbf{A}}$ associated with the numeriare $\ddot{a}_{x}$, such that all $\ddot{a}_{x^{-}}$ rebased price processes are martingales. Hence, also the price process for the forward annuity $r_{x}$ is a martingale under the measure $\mathbf{Q}^{\mathbf{A}}$.

Furthermore, the numeraire rebased market value $V^{G} / \ddot{a}_{x}$ of the GAO put-option must also be a martingale process under the probability measure $\mathbf{Q}^{\mathbf{A}}$. Using equation (2.4) which gives the value of the GAO put-option at time $T$, the value of the GAO option for any time $t \leq T$ can be expressed as

$$
\begin{aligned}
\frac{V^{G}(t)}{\ddot{a}_{x}(t)} & =\mathbf{E}^{A}\left[\frac{V^{G}(T)}{\ddot{a}_{x}(T)}\right]=\mathbf{E}^{A}\left[\frac{\max \left(r_{x}^{G}-r_{x}(T), 0\right) \ddot{a}_{x}(T)}{\ddot{a}_{x}(T)}\right] \\
& =\mathbf{E}^{A}\left[\max \left(r_{x}^{G}-r_{x}(T), 0\right)\right],
\end{aligned}
$$

where $\mathbf{E}^{\mathbf{A}}[]$ denotes an expectation under the probability measure $\mathbf{Q}^{\mathbf{A}}$. Multiplying both sides of equation (2.8) by $\ddot{a}_{x}(t)$ leads to the following expression for the market price of the GAO:

$$
V^{G}(t)=\ddot{a}_{x}(t) \mathbf{E}^{A}\left[\max \left(r_{x}^{G}-r_{x}(T), 0\right)\right]
$$

Once we know the probability distribution for $r_{x}(T)$ under the measure $\mathbf{Q}^{\mathbf{A}}$, we can use expression (2.9) to calculate the value of the GAO option explicitly. The market standard assumption for 
pricing interest rate options is to assume that the forward rate process has a lognormal probability distribution, which leads to the Black (1976) option pricing formula.

If we examine the definition (2.2) of the annuity payout rate $r_{x}(T)$ in more detail, we see that the payout rate decreases if the discount bond prices increase, i.e. if the market interest rates become lower. If the market interest rates all fall to zero, the discount bonds all have a market value equal to 1 and the insurance company can still offer a positive payout rate $r_{x} *$ to its policyholders:

$$
r_{x}^{*}=\frac{1}{\sum_{n=0}^{\omega-x}{ }_{n} p_{x}}
$$

With positive market interest rates, the annuity payout rate will always be higher than the minimum rate $r_{x} *$. Hence, a more realistic distributional assumption for the annuity payout rate $r_{x}(T)$ is the assumption that the shifted random variable $r_{x}(T)-r_{x} *$ has a lognormal distribution. Based on this shifted lognormal distribution, the price for the GAO put-option is given by

$$
\begin{aligned}
V^{G}(t) & =\dot{a}_{x}(t)\left(r_{x}^{G} N\left(-d_{2}\right)-r_{x}(t) N\left(-d_{1}\right)\right) \\
d_{1,2} & =\frac{\ln \left(\frac{r_{x}(t)-r_{x}{ }^{*}}{r_{x}^{G}-r_{x}{ }^{*}}\right) \pm \frac{1}{2} \sigma_{r}^{2}(T-t)}{\sigma_{r} \sqrt{T-t}}
\end{aligned}
$$

where $\sigma_{r}$ denotes the volatility of the (shifted lognormal) process $r_{x}(T)-r_{x} *$.

\section{Static Replicating Portfolio}

The GAO put-option we have discussed in the previous section, is not a standard interest rate option. To hedge the risk of such a non-standard option, an insurance company can execute a dynamic replication strategy (delta hedging). This replication strategy requires continuous rebalancing of a portfolio of discount bonds. Executing such a trading strategy in practice can be problematic, especially over the long time periods that are typically involved in life-insurance products.

We therefore want to propose a static options replication strategy that can be used to hedge the risk of GAO's. In a static options replication strategy one sets up a portfolio of actively traded 
options such that the payoff of the GAO at maturity is exactly replicated. Due to the fact that this portfolio matches the payoff of the GAO at maturity, the portfolio will also accurately track at all previous times the value of the GAO. Were this not the case, an arbitrage opportunity would arise. Hence, once the initial portfolio of options is bought, its composition never needs to be adjusted until the time that the GAO expires.

In the remainder of this section we show how a static replication portfolio of vanilla interest rate swaptions can be set up for GAO's. In interest rate markets, interest rate swaptions are the most actively traded options contracts and can be traded in large quantities for a wide variety of maturities and exercise prices. The construction we propose for GAO's is inspired by the static replication strategy proposed by Hunt and Kennedy (2000, Ch. 15) for irregular swaptions.

At the exercise date $T$, the GAO put-option gives the holder the right, but not the obligation, to enter into an annuity at the guaranteed rate $r^{G}$ :

$$
V^{G}(T)=\max \left(r_{x}^{G}-r_{x}(T), 0\right) \ddot{a}_{x}(T)=\max \left(\sum_{n=0}^{\omega-x}\left({ }_{n} p_{x} r_{x}^{G}\right) D_{T+n}(T)-1,0\right),
$$

where we have substituted the definition $\ddot{a}_{x}$ given in equation (2.1). Hence, the GAO gives the right to obtain a series of cash payments ${ }_{n} p_{x} r_{x}{ }^{G}$ at the different dates $T+n$ for the price of 1 at time $T$. Note that, due to the fact that the annuity payments are made at the beginning of each year, at time $T$ one has to pay 1 but one receives $r_{x}^{G}$ immediately so that the net cash flow at time $T$ is equal to $1-r_{x}^{G}$.

A vanilla interest rate swaption gives the right, but not the obligation, to enter at time $T$ into an interest rate swap in which during $N$ years the floating LIBOR interest rate is exchanged for a fixed interest rate $K_{N}$. It is well known that the market value $S^{N}$ of a receiver swap in which the fixed rate is received annually is given by (see, e.g. Hull (2000, Ch. 5))

$$
S^{N}(T)=\left(\sum_{n=1}^{N-1} K_{N} D_{T+n}(T)+\left(1+K_{N}\right) D_{T+N}(T)\right)-1
$$


Hence, the market value $V^{N}$ of a receiver swaption that gives the right to enter into an $N$-year receiver swap at time $T$ can be expressed as

$$
V^{N}(T)=\max \left(S^{N}(T), 0\right)=\max \left(\left(\sum_{n=1}^{N-1} K_{N} D_{T+n}(T)+\left(1+K_{N}\right) D_{T+N}(T)\right)-1,0\right)
$$

From expression (3.3) we see that, similar to the GAO, a swaption also gives the right to obtain a series of cash payments for a price of 1 . However, the pattern of the cash payments is very different in the two options. The cash flows ${ }_{n} p_{x} r_{x}{ }^{G}$ associated with the guaranteed annuity are gradually decreasing over time due to the survival probabilities ${ }_{n} p_{x}$. The cash flows associated with an $N$-year swap follow a very different pattern: the first $N$-1 years one receives an amount of $K_{N}$, whereas in the $N$ th year, a cash amount of $\left(1+K_{N}\right)$ is received.

By combining positions in receiver swap contracts all starting at date $T$ with different maturities $N$, it is possible to replicate the cash flow pattern ${ }_{n} p_{x} r_{x}^{G}$ of the guaranteed annuity for all dates $T+n$. To find the right amounts that has to be invested in each swap, we proceed backwards from time $T+(\omega-x)$ to time $T+1$. To replicate the cash flow $\omega_{x} p_{x} r_{x}{ }^{G}$ we have to enter into the $(\omega-x)$-year receiver swap $S^{\omega-x}$ with fixed rate $K_{\omega-x}$. At time $T+(\omega-x)$ this swap has a cash flow of $\left(1+K_{\omega-x}\right)$. Hence, if we invest an amount $L_{\omega-x}=\omega_{\omega-x} p_{x} r_{x}^{G} /\left(1+K_{\omega-x}\right)$ in swap $S^{\omega-x}$ we replicate the cash flow of the guaranteed annuity at time $T+(\omega-x)$.

One year earlier, at time $T+(\omega-x-1)$, the guaranteed annuity pays out a cash flow of $\omega_{-x-1} p_{x} r_{x}^{G}$. From the position $L_{\omega-x}$ in swap $S^{\omega-x}$ we already receive a cash flow of $K_{\omega-x} L_{\omega-x}={ }_{\omega-x} p_{x} r_{x}^{G}-L_{\omega-x}$. Hence, if we invest an amount $\left.L_{\omega-x-1}=\left(L_{\omega-x}+r_{x}{ }^{G}(\omega-x-1) p_{x}-\omega_{-x} p_{x}\right)\right) /\left(1+K_{\omega-x-1}\right)$ in swap $S^{\omega-x-1}$ we replicate the cash flow of the guaranteed annuity at time $T+(\omega-x-1)$.

Continuing this backward construction, we find that we can replicate the cash flow of the guaranteed annuity at a general date $T+n$ by investing an amount $L_{n}=\left(L_{n+1}+r_{x}^{G}\left({ }_{n} p_{x}-{ }_{n+1} p_{x}\right)\right) /$ $\left(1+K_{n}\right)$ in swap $S^{n}$. Proceeding backwards in this fashion, we continue to match all the cash payments of the guaranteed annuity up until time $T+1$.

However, there is a catch. From equation (3.2) we see that at the start date $T$ of the swap contract we require an initial cash payment of 1 . Hence, the total portfolio of receiver swaps constructed 
above to replicate the cash flows of the guaranteed annuity requires an initial cash payment of $\sum_{n=1}^{\omega-x} L_{n}$. But in equation (3.1) we derived that the GAO put-option gives the right to enter the guaranteed annuity for an initial net cash payment of $1-r_{x}{ }^{G}$. Fortunately, we can adjust the amounts $L_{n}$ by considering receiver swaps with different fixed rates $K_{n}$. This implies that we have to choose a set of fixed rates $K_{n}^{*}$ for all the swaps $S^{n}$ such that the invested amounts $L_{n}{ }^{*}$ satisfy $\sum_{n=1}^{\omega-x} L_{n}^{*}=1-r_{x}^{G}$

With the portfolio of swaps $\Sigma L_{n} * S^{n}$ we have replicated all the cash flows of the guaranteed annuity with rate $r_{x}^{G}$. Hence, the GAO which gives the right, but not the obligation, at time $T$ to enter into the guaranteed annuity is equivalent to the option to enter into the portfolio $\Sigma L_{n} * S^{n}$. This implies that the value $V^{G}(T)$ at time $T$ of the GAO can be expressed in terms of swaptions $V^{n}$ as:

$$
V^{G}(T)=\max \left(\sum_{n=1}^{\omega-x} L_{n}^{*} S^{n}(T), 0\right) \leq \sum_{n=1}^{\omega-x} L_{n}^{*} \max \left(S^{n}(T), 0\right)=\sum_{n=1}^{\omega-x} L_{n}^{*} V^{n}(T)
$$

where the inequality stems from the fact that the value an option on a portfolio of swaps is less than or equal to the value of the portfolio of the corresponding swaptions. An intuitive explanation for this fact is that in the option on the portfolio you have only an "all-or-nothing" choice to obtain all underlying swaps at once or none at all, whereas in the portfolio of swaptions you can "cherry pick" the individual swaps that have positive market values at time $T$.

If all the interest rates in the economy are perfectly correlated, i.e. all interest rates move all the time in perfect lockstep, then there exists only one single set of market swap rates $K_{n}{ }^{*}$ for which the swaps $S^{n}$ exactly replicate the cash flow stream of the guaranteed annuity. Due to the perfect correlation of the interest rates, all market swap rates will either be simultaneously above the rates $K_{n} *$ or simultaneously below. Hence, in the case of perfectly correlated interest rates, the inequality in equation (3.4) becomes an equality for the set of swaptions with strikes $K_{n} *{ }^{3}$ But this

\footnotetext{
${ }^{3}$ This remarkable result was derived for the first time by Jamshidian (1989) where he showed that in a one-factor interest rate model an option on a coupon bearing bond can be expressed as a
} 
implies that in the case of perfectly correlated interest rates, we have replicated the payoff of the GAO via a portfolio of vanilla interest rate swaptions and, a fortiori, that we have identified a static options replication for the GAO.

In practice we know that the interest rates in the economy are not perfectly correlated, and therefore that the portfolio of swaptions has a higher price than the GAO due to the inequality in equation (3.4). However, GAO's typically are products with a very long maturity. Therefore, their value depends mainly on the behaviour of interest rates with long maturities and these interest rates are very highly correlated. We therefore conjecture that the price of the static hedge replication will be very close to the true price of the GAO.

\section{Historical Test}

To test the performance of the static replication strategy we have proposed in Section 3, we have conducted an historical test using UK interest rate data. We have downloaded from Datastream UK Government Bond yields with maturities 2, 3, 5, 7, 10, 15, 20 and 30 years. We have used the data at the last trading day of each year from 1980 until 2000. In each year we used a NelsonSiegel (1987) parameterisation of the zero-curve. In each year the Nelson-Siegel parameters were obtained by a least squares fit of the yields implied by the zero-curve to the observed yields. The results of the parameter fits are reported in Table 1. (Table can be found at the end of this paper.) Note that, in order to stress-test our static hedge, we have also allowed the "time-scale" parameter tau to vary over time, to obtain as much as possible variation in the interest rates with long maturities. Practitioners usually keep the value of tau constant to stabilise the long end of the yield curve.

Given the Nelson-Siegel parameterisation, we have zero-rates available for all possible maturities. Using the mortality table GBM8590 from the Dutch Actuarial Institute, we can determine the forward annuity rate using formula (2.7). In Figure 1 below, we have plotted the forward annuity rates for a male that was 45 years old in 1980 and that would retire at age 65 in 2000. Initially, the forward annuity rate is well above the guaranteed level of $11.1 \%$. However, due to the falling interest rates at the end of the 1990's, we see that the forward annuity rate drops below the guaranteed level after 1997.

portfolio of options on zero coupon bonds. Note also, that in the case of perfectly correlated rates 


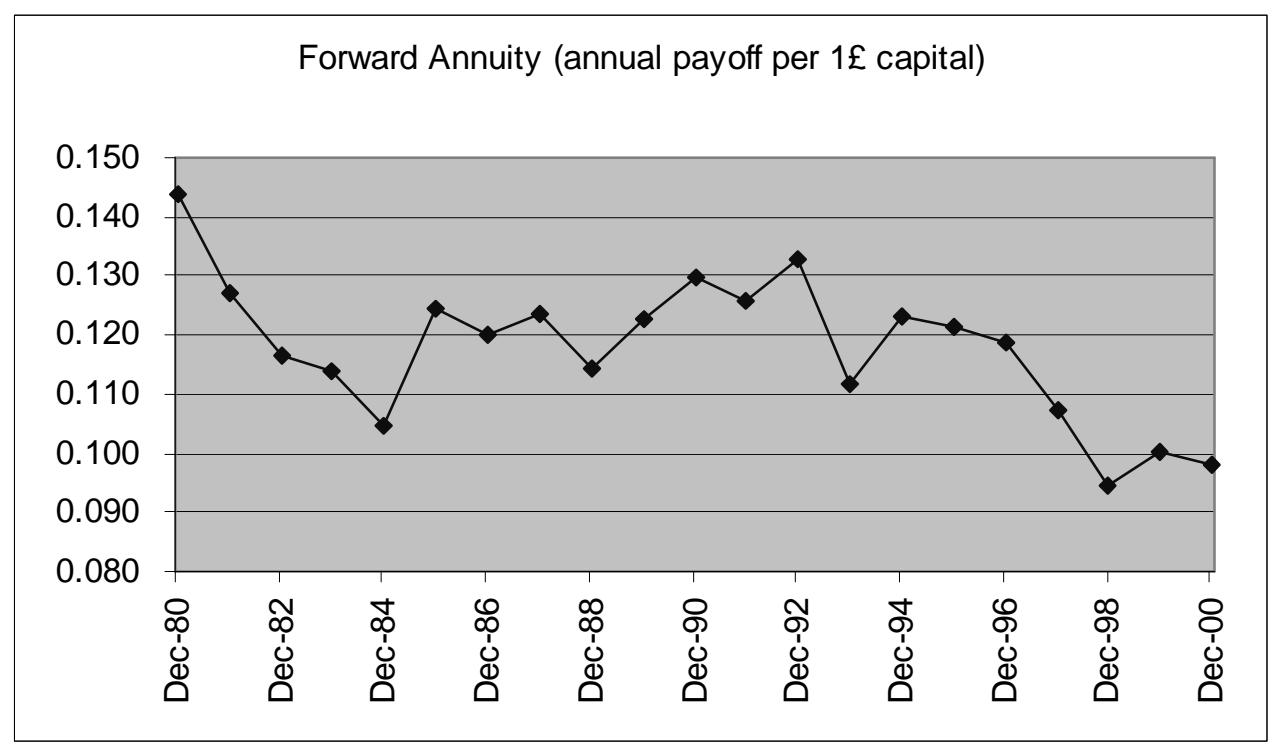

Figure 1: Forward annuity rate for UK data and Dutch mortality table

From the mortality table, we calculate that the minimum annuity rate $r_{65} *$ is equal to $6.78 \%$. From the time-series of the forward annuity rates, we estimate the volatility of the (shifted lognormal) forward annuity rate process at $21.22 \%$. Substituting the volatility in formula (2.11), we can calculate the market value of the GAO put-option.

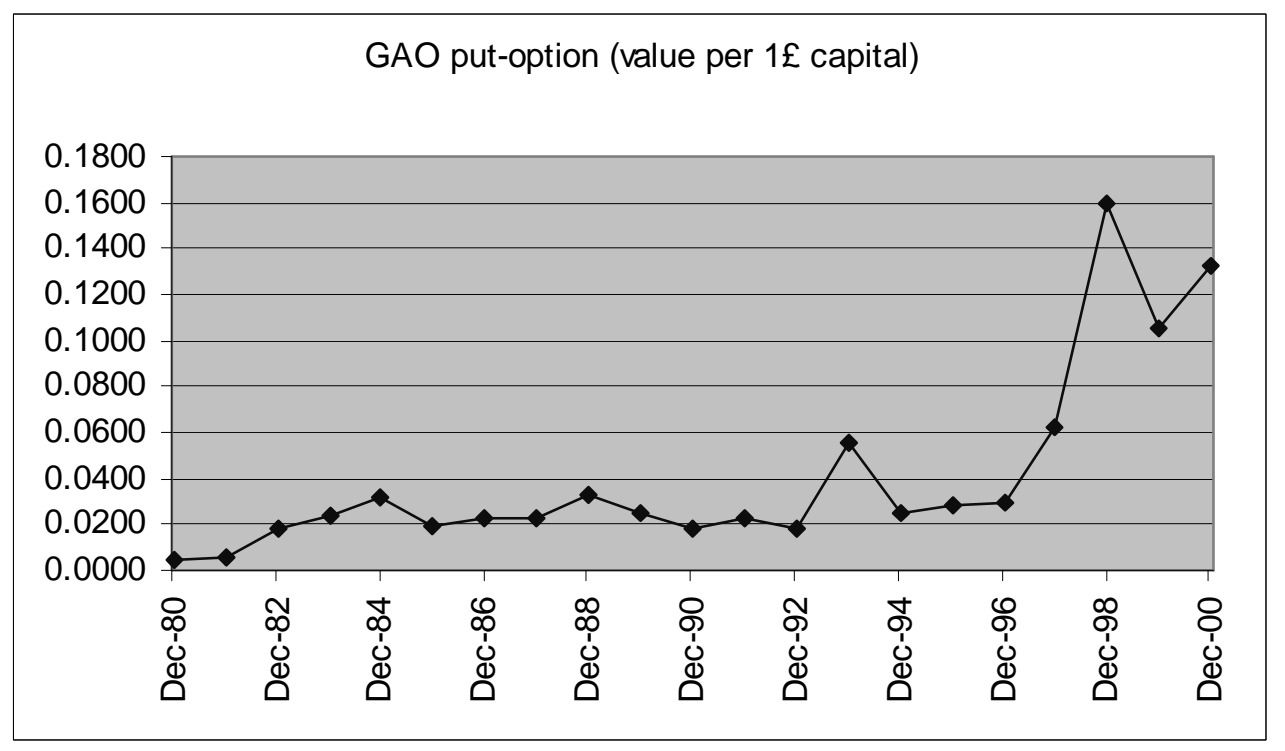

Figure 2: Market value of GAO put-option.

the apparent ambiguity in choosing the rates $K_{n} *$ is resolved. 
The calculated market values of the GAO put-option have been plotted in Figure 2. Again, we see that the value of the GAO put-option increases dramatically in value with the falling interest rates during the late 1990's. In fact, the value of the GAO increases almost a factor 30: from $0.45 \%$ in 1980 until 13.22\% in December 2000.

This already indicates what the disadvantages are of "only" reserving for maturity guarantees instead of replication: reserving is very expensive and does not give complete protection. See, for example, the results reported by Yang (2001). Yang calculates, on the basis of the Wilkie model, that the reserve at a $99 \%$ level that would have to be set aside in 1980 for the policy with term 20 is equal to $12.06 \%$. As Yang shows in Table 10 of his paper, the actual value of the GAO at the end of the 20-year period is even higher than this $99 \%$ reserve. Hence, even reserving at a $99 \%$ probability-level would not have provided sufficient protection against the explosive growth in value of the GAO put-option during the 20 year period from 1980 until 2000.

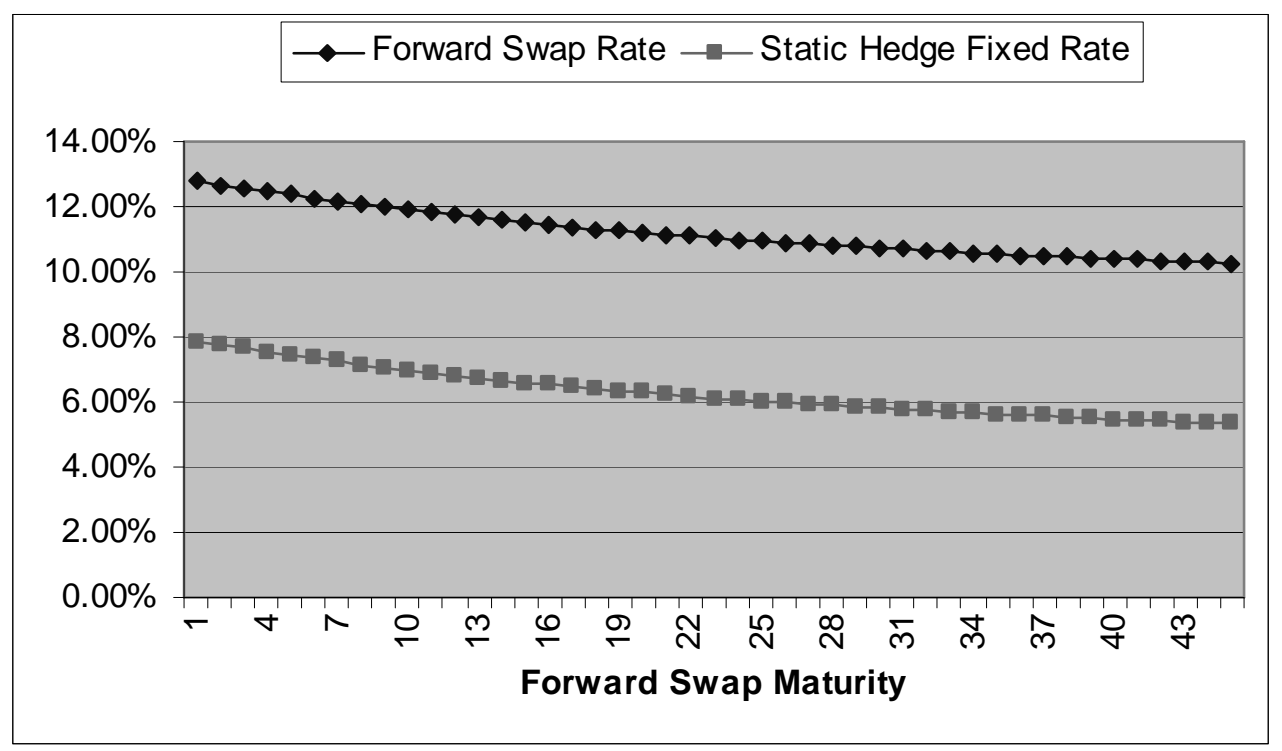

Figure 3a: Forward swap rates and static hedge fixed rates in Dec-1980

Setting up the static replication portfolio of vanilla swaptions is considerably cheaper than "only" reserving, and does provide better protection. In 1980, the insurance company should have forecasted the annuity payments for a then 45 year old person which would reach the retirement age 65 in the year 2000. In Figure 3a we have plotted the forward swap rates the prevailed in Dec1980. All swap rates are 20 year forward rates, with various swap maturities. We see that the forward swap rates slowly decrease from $12.79 \%$ for the 20-year forward 1-year swap rate, until $10.25 \%$ for the 20 -year forward 45 -year swap rate. 
As was explained in Section 3, to set up the static replicating portfolio, we have to select a set of fixed rates $K_{n} *$. If all the interest rates are correlated perfectly, this will be the swap rates for which the GAO will be exactly "at-the-money". To construct the static hedge portfolio, we have made the assumption that all interest rates are perfectly correlated and also that all interest rates move exactly parallel. Hence, we have shifted all the rates by the same amount until the invested amounts $L_{n} *$ satisfied $\sum_{n=1}^{45} L_{n}^{*}=1-0.111=0.889$. We found that this was achieved for a downward shift of $4.92 \%$-point. The set of fixed rates $K_{n} *$ obtained by this parallel shift of the swap rates has also been depicted in Figure 3a.

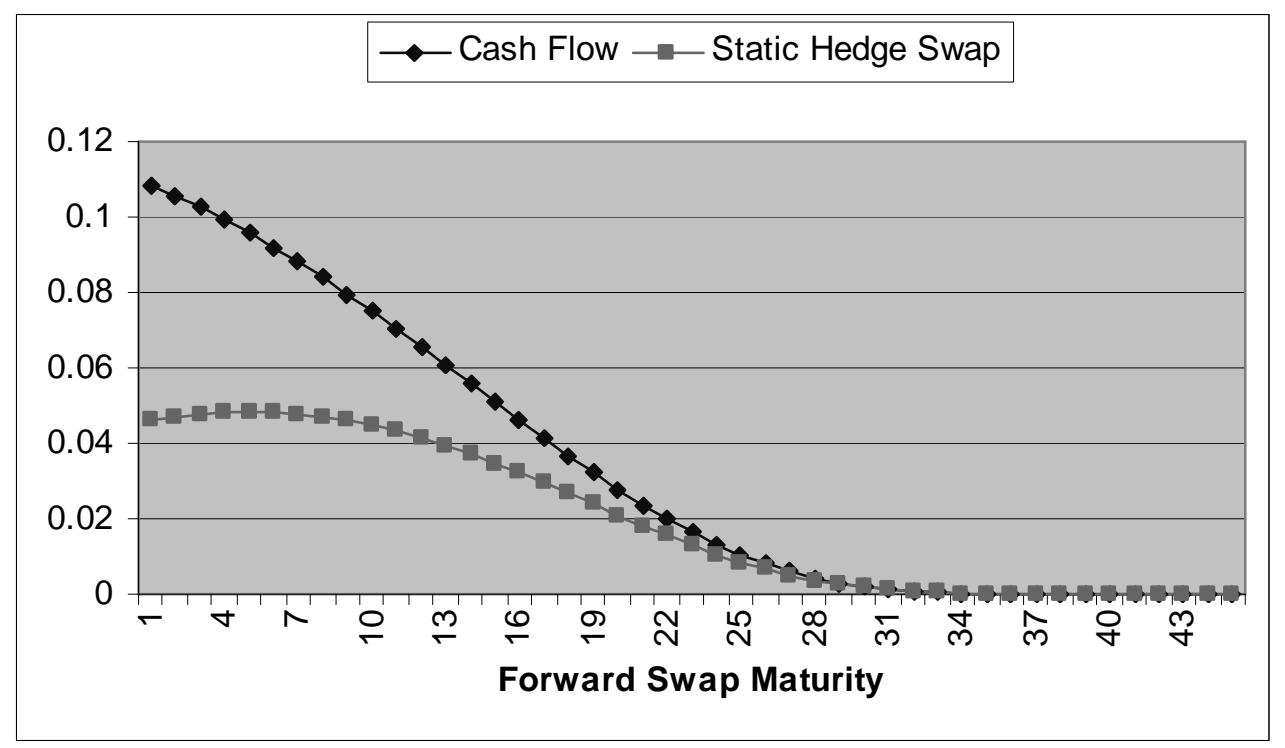

Figure 3b: Static Replication Portfolio of Annuity cash flows

In Figure 3b, we have plotted the projected cash flows for the annuity for the years 2001 until 2045. Also, we have plotted the weights $L_{n} *$ that have to be invested in all the swaps with fixed rates $K_{n} *$ for $n=1$ to 45 . Hence, with the weights $L_{n} *$ the insurance company could have bought the portfolio of vanilla swaptions $\Sigma_{n} L_{n} * V^{n}$. This portfolio of swaptions would cost 0.0047 per $1 £$ capital in 1980, which is only 0.0002 per $1 £$ capital more expensive than the true market value of the GAO put-option. Once this portfolio of swaptions would have been attained, no further buying or selling would have been necessary until December 2000, when the portfolio would have been unwound to cover the cost of the GAO put-option. 


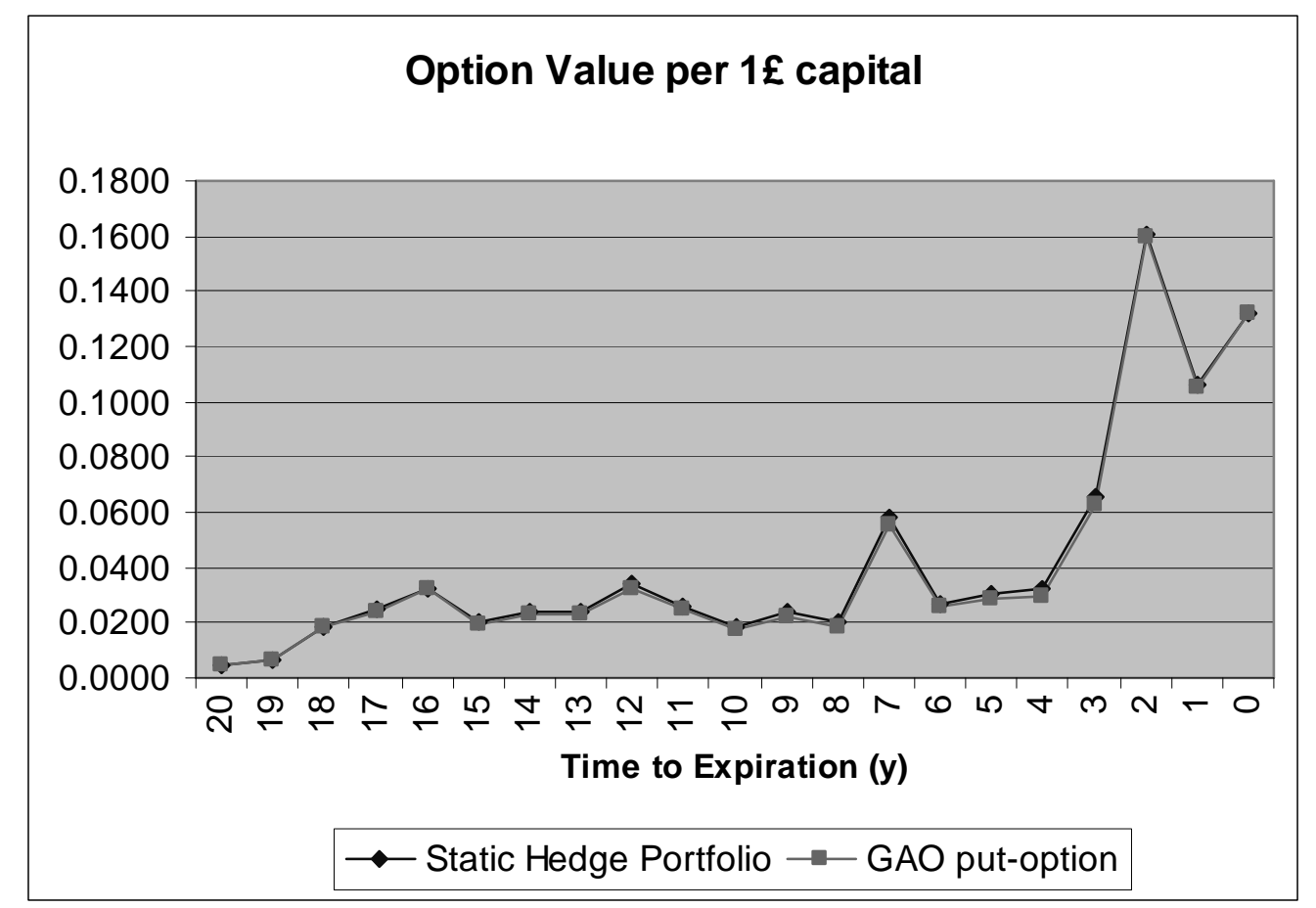

Figure 4: Performance of Static Hedge Portfolio vs. GAO put option

In Figure 4 we have plotted the value of the static replicating portfolio against the market value of the GAO put option for the period Dec-1980 until Dec-2000. The blue and purple lines depict the market value per $1 £$ capital of the static replicating portfolio and the market value of the GAO putoption respectively. We see that the value of the static replicating portfolio tracks the market value of the GAO extremely closely during the whole period of 20 years.

\section{Summary and Conclusion}

In this paper we have derived a market value for Guaranteed Annuity Option using martingale modelling techniques. Furthermore, we have shown how to construct a static replicating portfolio of vanilla swaptions that replicates the Guaranteed Annuity Option. Finally, we have shown with historical UK interest rate data from 1980 until 2000 that the static replicating portfolio is extremely effective as a hedge against the interest rate risk involved in the GAO, and that the static replicating portfolio is considerably cheaper than up-front reserving and also that the replicating portfolio provides a much better level of protection than a fixed reserve. 
Table 1: Nelson-Siegel zero-curves

\begin{tabular}{|r|rrrr|}
\hline & beta0 & beta1 & beta2 & tau \\
\hline $\mathbf{1 2} / \mathbf{3 1 / 8 0}$ & 0.0000 & 0.1255 & 0.2242 & 20.2 \\
$\mathbf{1 2} / \mathbf{3 1 / 8 1}$ & 0.0000 & 0.1412 & 0.2675 & 12.0 \\
$\mathbf{1 2 / 3 1 / 8 2}$ & 0.0374 & 0.0622 & 0.1396 & 10.0 \\
$\mathbf{1 2 / 3 0 / 8 3}$ & 0.0649 & 0.0269 & 0.1068 & 5.0 \\
$\mathbf{1 2 / 3 1 / 8 4}$ & 0.0291 & 0.0669 & 0.1696 & 7.0 \\
$\mathbf{1 2 / 3 1 / 8 5}$ & 0.0873 & 0.0295 & 0.0275 & 3.0 \\
$\mathbf{1 2 / 3 1 / 8 6}$ & 0.0566 & 0.0524 & 0.0582 & 10.0 \\
$\mathbf{1 2 / 3 1 / 8 7}$ & 0.0417 & 0.0452 & 0.0993 & 12.7 \\
$\mathbf{1 2 / 3 0 / 8 8}$ & 0.0531 & 0.0628 & 0.0243 & 10.0 \\
$\mathbf{1 2} / \mathbf{2 9 / 8 9}$ & 0.1059 & 0.0252 & -0.0852 & 10.0 \\
$\mathbf{1 2 / 3 1 / 9 0}$ & 0.0845 & 0.0324 & 0.0095 & 10.0 \\
$\mathbf{1 2 / 3 1 / 9 1}$ & 0.0878 & 0.0100 & 0.0238 & 3.0 \\
$\mathbf{1 2 / 3 1 / 9 2}$ & 0.1005 & -0.0139 & -0.0867 & 1.6 \\
$\mathbf{1 2 / 3 1 / 9 3}$ & 0.0657 & -0.0256 & 0.0252 & 4.1 \\
$\mathbf{1 2 / 3 0 / 9 4}$ & 0.0806 & -0.0123 & 0.0430 & 3.0 \\
$\mathbf{1 2 / 2 9 / 9 5}$ & 0.0644 & -0.0087 & 0.0643 & 10.0 \\
$\mathbf{1 2 / 3 1 / 9 6}$ & 0.0778 & -0.0195 & 0.0157 & 3.0 \\
$\mathbf{1 2 / 3 1 / 9 7}$ & 0.0616 & 0.0106 & -0.0064 & 3.0 \\
$\mathbf{1 2 / 3 1 / 9 8}$ & 0.0440 & 0.0224 & -0.0252 & 1.5 \\
$\mathbf{1 2 / 3 1 / 9 9}$ & 0.0367 & 0.0201 & 0.0552 & 2.3 \\
$\mathbf{1 2 / 2 9 / 0 0}$ & 0.0241 & 0.0293 & 0.0233 & 10.0 \\
\hline & & & & \\
\hline
\end{tabular}




\section{References}

K. Aase and S. Persson, (1994), "Pricing of Unit-linked Life Insurance Policies," Scandianavian Actuarial Journal, Vol. 1, 26-52.

K. Aase and S. Persson, (1997), "Valuation of the Minimum Guaranteed Return Embedded in Life Insurance Products", Journal of Risk and Insurance, Vol. 64(4), 599-617.

A. Bacinello and S. Persson, (2002), "Design and Pricing of Equity-Linked Life Insurance under Stochastic Interest Rates,” Journal of Risk Finance, Vol. 3(2), 6-21.

L. Ballotta and S. Haberman, (2002), "Valuation of Guaranteed Annuity Conversion Options," Working paper, City University, London.

F. Black, (1976), “The pricing of Commodity Contracts," Journal of Financial Economics, Vol. 3, 167-179.

F. Black and M. Scholes, (1973), "The pricing of Options and Corporate Liabilities," Journal of Political Economy, Vol. 81(3), 637-654.

P. Bouwknegt and A. Pelsser, (2002), "Market Value of Profit-sharing," Journal of Risk Finance, forthcoming.

N. Bowers, H. Gerber, J. Hickman, D. Jones and C. Nesbitt, (1997), Actuarial Mathematics (2 ${ }^{\text {nd }}$ $E d$.), The Society of Actuaries, Illinois.

P. Boyle and M. Hardy, (1997), "Reserving for Maturity Guarantees: Two Approaches," Insurance: Mathematics and Economics, Vol. 21, 113-127.

P. Boyle and E. Schwartz, (1977), "Equilibrium prices of equity linked insurance policies with an asset value guarantee," Journal of Risk and Insurance, Vol. 44, 639-660.

A. Brace, D. Gatarek and M. Musiela, (1997), "The Market Model of Interest Rate Dynamics," Mathematical Finance, Vol. 7, 127-154. 
M. Brennan and E. Schwartz, (1976), "The pricing of equity-linked life insurance policies with an asset value guarantee," Journal of Financial Economics, Vol. 3, 195-213.

P. Carr, K. Ellis and V. Gupta, (1998), "Static Hedging of Exotic Options," Journal of Finance, Vol. 53(3), 1165-1190.

E. Derman, D. Ergener and I. Kani, (1995), "Static Options Replication," The Journal of Derivatives, Vol. 2(4), 78-95.

A. Grosen and P. Jørgensen, (1997), "Valuation of Early Exercisable Interest Rate Guarantees," Journal of Risk and Insurance, Vol. 64 (3), 481-503.

A. Grosen and P. Jørgensen, (2000a), "Fair valuation of life insurance liabilities: The impact of interest rate guarantees, surrender options, and bonus policies," Insurance: Mathematics and Economics, Vol. 26, 37-57.

A. Grosen and P. Jørgensen, (2002), “Life Insurance Liabilities at Market Value,” Journal of Risk and Insurance, forthcoming.

D. Heath, R. Jarrow and A. Morton, (1992), "Bond Pricing and the Term Structure of Interest Rates: A New Methodology for Contingent Claims Valuation,” Econometrica, Vol. 60(1), 77-105.

J. Hull, (2000), Options, Futures, and Other Derivatives ( ${ }^{\text {th }}$ Ed.), Prentice-Hall, New Jersey.

P. Hunt and J. Kennedy, (2000), Financial Derivatives in Theory and Practice, Wiley and Sons, Chichester.

F. Jamshidian, (1989), “An Exact Bond Option Formula,” Journal of Finance, Vol. 44, 205-209.

F. Jamshidian, (1998), "LIBOR and Swap Market Models and Measures," Finance and Stochastics, Vol. 1(4), 293-330. 
P. Lee, (2001), "Pricing and Reserving for Guaranteed Annuity Options," presentation for Den Danske Aktuarforening, downloadable from http://www.inqa.com/Conferences.htm.

K. Miltersen and S. Persson, (1999), "Pricing Rate of Return Guarantees in a Heath-JarrowMorton Framework," Insurance: Mathematics and Economics, Vol. 25(3), 307-325.

K. Miltersen and S. Persson, (2002), "Guaranteed Investment Contracts: Distributed and Undistributed Excess Return," Scandinavian Actuarial Journal, forthcoming.

K. Miltersen, K. Sandmann and D. Sondermann, (1997), "Closed Form Solutions for Term Structure Derivatives with Lognormal Interest Rates," Journal of Finance, Vol. 52, 409-430.

M. Musiela and M. Rutkowski, (1997), Martingale Methods in Financial Modelling, Springer Verlag, Berlin.

C. Nelson and A. Siegel, (1987), "Parsimonious Modelling of Yield Curves," Journal of Business, Vol. 60(4), 473-489.

A. Pelsser, (2000), Efficient Methods for Valuing Interest Rate Derivatives, Springer Verlag, Berlin.

S. Yang, (2001), "Reserving for Guaranteed Annuity Options: A Stochastic Simulation Approach," Working Paper, Heriot-Watt University, Edinburgh. 